P. Nau, J. Koppmann, A. Lackner, A. Brockhinke, Detection of formaldehyde in flames using UV and MIR absorption spectroscopy, Z. Phys. Chem. 229 (2014) 483-494.

This paper was published in Zeitschrift für Physikalische Chemie and is made available as an electronic reprint with the permission of Oldenbourg Wissenschaftsverlag. 


\title{
Detection of formaldehyde in flames using UV and MIR absorption spectroscopy
}

\author{
Patrick Nau ${ }^{1,2}$, Julia Koppmann ${ }^{2}$, Alexander Lackner ${ }^{2}$, Andreas \\ Brockhinke $^{2}$ \\ 1 German Aerospace Center, Institute of Combustion Technology, Pfaffen- \\ waldring 38-40, 70569 Stuttgart \\ ${ }^{2}$ Universität Bielefeld, Physikalische Chemie 1, Universitätsstraße 25, 33615 \\ Bielefeld
}

Received: date / Revised version: date

\begin{abstract}
Absorption spectroscopy in the ultraviolet (UV) and midinfrared (MIR) spectral region has been used in a comparative study for the detection of formaldehyde in laminar low pressure flames of dimethylether (DME) and methane. Both spectral regions were tested to explore respective advantages and limitations, especially for the detection of stable molecules in flames. In the UV, cavity ring-down spectroscopy (CRDS), a highly sensitive multi-pass absorption technique, has been used for the detection of formaldehyde in the $A-X 4_{1}^{0}$ hot band at $370 \mathrm{~nm}$. For measurements in the mid-infrared, the fundamental CO-stretch vibration $\nu_{2}$ of formaldehyde at $5.9 \mu \mathrm{m}$ was used. MIR laser radiation was generated with a pulsed quantum cascade laser (QCL), a novel laser type which offers high output energies and excellent beam quality in the MIR. It was shown that MIR-QCL absorption spectroscopy and UV-CRDS have a comparable sensitivity, even though only single-pass absorption was used in the MIR. Additionally, MIRQCL is clearly superior in terms of the simplicity of the experimental setup. This shows the large potential of QCL based laser absorption spectroscopy for the sensitive detection of trace species in the MIR.
\end{abstract}

\section{Introduction}

Formaldehyde is an important intermediate in combustion chemistry and also a toxic product of incomplete combustion. It has been shown that significant amounts of formaldehyde are produced in the combustion of oxygenated fuels such as ethers and alcohols. This is a pressing problem, since

Correspondence to: Andreas Brockhinke, +49-521-106 2189,
andreas.brockhinke@uni-bielefeld.de 
most biofuels which are developed as alternative to hydrocarbons are of this type[1]. In combustion studies, formaldehyde has frequently been detected by absorption or laser-induced fluorescence (LIF) measurements in the UV after excitation of the $A-X$ transition, which is readily accessible with tripled Nd:YAG laser $(355 \mathrm{~nm})$ or XeF Excimer laser $(353 \mathrm{~nm})$ radiation $[2-7]$. To reduce the influence of the highly temperature sensitive absorption cross section in this region, other researchers used the hot band $4_{1}^{0}$ at $370 \mathrm{~nm}$ instead $[8,9]$. One drawback of all investigations in the UV is underlying broadband absorption which complicates precise quantification in many cases. Additionally, fluorescence from polycyclic aromatic hydrocarbons (PAH) and Raman scattering can contribute to the detected signal[7]. Sampling probe measurements combined with mass spectrometry[10] or diode laser absorption[11] have been reported as well. This is, however, not a generally applicable approach due to the complicated setup and since the influence of the sampling probe on flame chemistry is still under discus$\operatorname{sion}[12]$.

An alternative to investigations in the UV are measurements in the MIR spectral range. Recently, Wang et al. used a cw DFG (difference frequency generation) laser in the $3.6 \mu \mathrm{m}$ region ( $\nu_{1}$ and $\nu_{5}$ fundamental band) to detect formaldehyde in a shock tube[13]. Even better suited for diagnostic purposes is the strong fundamental CO-stretch vibration $\nu_{2}$ in the $5.7 \mu \mathrm{m}$ region. Up to now, measurements in this region have been scarce due to the lack of suitable light sources in this spectral region. This has been changed, however, with the advent of quantum cascade lasers (QCL). Due to their high output power and high spectral resolution, QCL are suited well for spectroscopic measurements and have been used in several recent studies of environmental research[14,15], plasma measurements[16] and combustion chemistry[17-19].

In our work, we compare two different absorption techniques for the quantitative detection of formaldehyde in low pressure flames and discuss their respective merits. For absorption measurements in the UV, cavity ringdown spectroscopy (CRDS) utilising a pulsed dye laser has been used. This technique enables high sensitivity due to multiple reflections of the laser beam within an optical cavity. In the MIR, absorption at $5.9 \mu \mathrm{m}$ has been used. This transition involves the strong fundamental CO-stretch vibration $\nu_{2}$. Due to the much simpler spectrum in the MIR compared to the UV, underlying broadband absorption and interferences with other molecules can be completely avoided. Tunable MIR laser radiation is generated with a pulsed distributed feedback (DFB) quantum cascade laser which can be chirped over $2 \mathrm{~cm}^{-1}$ within a single laser pulse. Therefore, and because of the strong transitions involved, this laser is especially well suited for direct laser absorption spectroscopy (LAS). 


\section{Experiment and data analysis}

Laminar, premixed, flat fuel/oxygen/argon (25\% argon) flames have been stabilized on a home-made sintered bronze matrix burner. The diameter of the central matrix is $66 \mathrm{~mm}$ diameter surrounded with a $20 \mathrm{~mm}$ coflow matrix ring. The burner can be moved with stepper motors horizontally and vertically inside a low pressure chamber. For the QCL measurements a fuel rich $(\phi=1.86)$ dimethylether (DME) flame has been investigated at $33 \mathrm{mbar}$ with a cold gas velocity at $300 \mathrm{~K}$ of $0.78 \mathrm{~m} / \mathrm{s}$. CRDS measurements were performed in a fuel rich $(\phi=1.8)$ methane flame (cold gas velocity at $300 \mathrm{~K}$ : $0.52 \mathrm{~m} / \mathrm{s}$ ). Gas flows were regulated by calibrated mass flow controllers. Both flames had similar peak temperatures $(2400 \mathrm{~K}$ for the DME flame and $2150 \mathrm{~K}$ for the methane flame).

\subsection{QCL experiment}

The QCL (Cascade Technologies, LS-03-D) is stabilized at a base temperature of $30^{\circ} \mathrm{C}$ and driven at $20 \mathrm{kHz}$ with a $500 \mathrm{~ns}$ long rectangular current pulse. This leads to a frequency downchirp of about $2 \mathrm{~cm}^{-1}$ and allows to record a complete absorption spectrum during each laser pulse. Since this frequency downchirp is nonlinear, a Ge-Etalon (with a free spectral range of $0.048 \mathrm{~cm}^{-1}$ ) is used to calibrate the spectral axis. Absolute wavenumbers are obtained by comparing the recorded spectrum with the known line positions of water[20] and formaldehyde[21] in the HITRAN/HITEMP database.

The MIR laser beam is focused into the center of the low pressure chamber with a $\mathrm{CaF}_{2}$ lens $(f=+500 \mathrm{~mm})$. This leads to a spatial resolution of about $0.4 \mathrm{~mm}$ in the burner center and has been checked with the knife edge technique. To reduce reflection losses and avoid etaloning, the windows of the chamber are mounted in Brewster's angle. Inside the burner housing, the laser beam is guided through rods (inner diameter $5 \mathrm{~mm}$ ) with a small exit hole (diameter $1 \mathrm{~mm}$ ) close to the burner. A weak nitrogen gas flow $(0.02 \mathrm{slm})$ avoids accumulation of stable combustion products into the rods and reduces its contribution to the measured absorption signal. Behind the burner chamber the laser pulse is detected with a fast MCT detector (Vigo Systems, PVI-2TE-8) and digitized with a fast digitizer (Agilent-Acqiris, U1071A-002, 8 bit, $2 \mathrm{GS} / \mathrm{s}$ ). The detector is AC coupled with a low cut-off frequency of $1 \mathrm{kHz}$. Therefore, any $\mathrm{cw}$ signals (e.g. originating from flame luminosity) are effectively suppressed.

The absorption spectrum is calculated from the natural logarithm of the ratio of absorption measurement and baseline measurement (recorded in regular intervals in the co-flow region next to the flame). Even though recording a baseline spectrum is not strictly necessary for data evaluation, this procedure compensates for the characteristic structure visible especially at the beginning of the laser pulse. Absorption spectra of 20,000 laser pulses are averaged to increase the signal-to-noise ratio. Tomographic reconstruction is used to obtain the absorption spectrum at a given point in the 
flame. For this purpose the burner is moved horizontally in steps of $1.5 \mathrm{~mm}$ from the flame center to the gas surrounding the flame. For data evaluation the Three-Point Abel Deconvolution of Dasch[22,23] is used. Absolute concentrations are obtained by fitting simulated absorption spectra to the Abel-inverted spectra with a least squares fitting algorithm based on the spectroscopic constants of the HITRAN/HITEMP database[21,20]. For the lines used in our data evaluation, the accuracy of the absorption cross sections is $5-10 \%[20]$. According to Beer-Lambert's Law, the absorption $\alpha$ of a line of the molecule $m$ is determined by the absorption cross section $\sigma$, the path length $d$, and the number density $N$. Finally, the complete absorption spectrum is determined by adding the absorption of every line $i$ at a given wavenumber:

$$
\frac{\alpha}{d}=\sum^{m} \sum^{i} \sigma_{i}(\nu) \times N_{m}
$$

$\sigma(\nu)$ can be calculated using the integrated absorption cross section $\sigma$ and the lineshape function $g(\nu)$ using $\sigma(\nu)=g(\nu) \times \sigma$. In our case, $g(\nu)$ can be well described by a Voigt function[24] with the Doppler broadening being the dominant contribution in the low pressure flames investigated here and pressure broadening playing only a minor role. More details of the experimental setup and the data evaluation procedure for the QCL measurements may be found in a recent publication[25].

\subsection{CRDS experiment}

Absorption spectra in the UV were measured with cavity ring-down spectroscopy (CRDS) utilizing a tunable dye laser setup. The dye laser is pumped with a Nd:YAG-laser (Spectra Physics, Lab150-10) using Styryl 8 dye dissolved in methanol. After frequency doubling, tunable UV radiation between $360-380 \mathrm{~nm}$ is generated. To improve mode matching in the CRD cavity, the laser beam passes a 2:1 telescope with an $100 \mu \mathrm{m}$ aperture in the focus of the first lens. The laser beam is then coupled into the cavity inside the low pressure chamber. The cavity consists of two dielectric mirrors $(R=99.98 \%)$ mounted on Pico motor actuated mirror mounts (NewFocus, 8807). Light exiting the cavity is detected with a photomultiplier (Philips, XP2020-Q) and recorded with an oscilloscope (GaGe, CompuScope12400). Ring-down traces are stored and evaluated on a personal computer with a program written in LabVIEW.

The intensity of the light exiting the ring-down cavity decays due to absorption, scattering and mirror losses. In case of a monochromatic laser pulse, the decay can be described with a monoexponential ring-down curve.

$$
\tau=\frac{L}{c(\Gamma+(1-R)+\sigma(\nu) N d))}
$$




$$
\sigma(\nu) N d=\left(\frac{1}{\tau}-\frac{1}{\tau_{0}}\right) \frac{L}{c}
$$

where $L$ is the length of the cavity and $(1-R)$ are the mirror losses. The parameter $\Gamma$ includes all additional losses such as broad band absorption or scattering. $\tau_{0}$ is the off-resonance ring-down time and leads to a baseline in the measured spectrum. It depends on $(1-R)$ and $\Gamma$, but changes only slowly with the wavelength. For the quantitative data evaluation, $\tau_{0}$ has been determined using a flat part of the spectrum next to the formaldehyde resonances. To obtain the decay rate of the ring-down traces, the curves were fitted between 90 and $10 \%$ of the peak intensity with a monoexponential least squares fit.

\section{Results}

With the dye laser/dye combination used, the complete formaldehyde $A-X 4_{1}^{0}$ band between 26600 and $27450 \mathrm{~cm}^{-1}$ could be measured (Fig. 1). A significant underlying broadband absorption is clearly visible. Even at the band heads, it contributes up up to $30 \%$ to the measured peak absorption. Careful subtraction of this offset is mandatory, both for absorption and LIF measurements, if quantitative concentration results are desired. The spectral range selected in our work covers the hot band of formaldehyde. This significantly reduces the influence from the highly temperature sensitive absorption cross section that complicates measurements after excitation of the ground state[9].

In most publications reporting LIF measurements of formaldehyde in flames, only relative concentrations are given. The major reason is that quantification of LIF is a complicated feat, especially in the case of formaldehyde $[27,3,2,6]$. One exception is the paper of Shin et al. who used a static cell at room temperature with a known concentration to calibrate the LIF measurements[28]. The measurement error was reported to be $30 \%$ and was said to be dominated by the temperature uncertainties. However, several additional sources for systematic errors can be present in a flame environment. One aspect which has to be taken into account is the broadband background of formaldehyde at high temperatures mentioned above. In addition, other broadband spectral features (e.g. from PAH fluorescence) might contribute to the total spectrum, especially at higher pressures. To reduce the influence from PAH, high power lasers can be used, since PAH fluorescence saturates at lower laser energies compared to formaldehyde[7]. Furthermore, Raman scattering from hydrocarbons must be considered for LIF measurements, since these signals might interfere with the desired signal. Quantitative LIF measurements also require knowledge of the quenching rates, which depend on both the gas composition and the temperature. Information on these energy transfer processes for formaldehyde in the UV is scarce; therefore, the accuracy of LIF measurements is limited. 


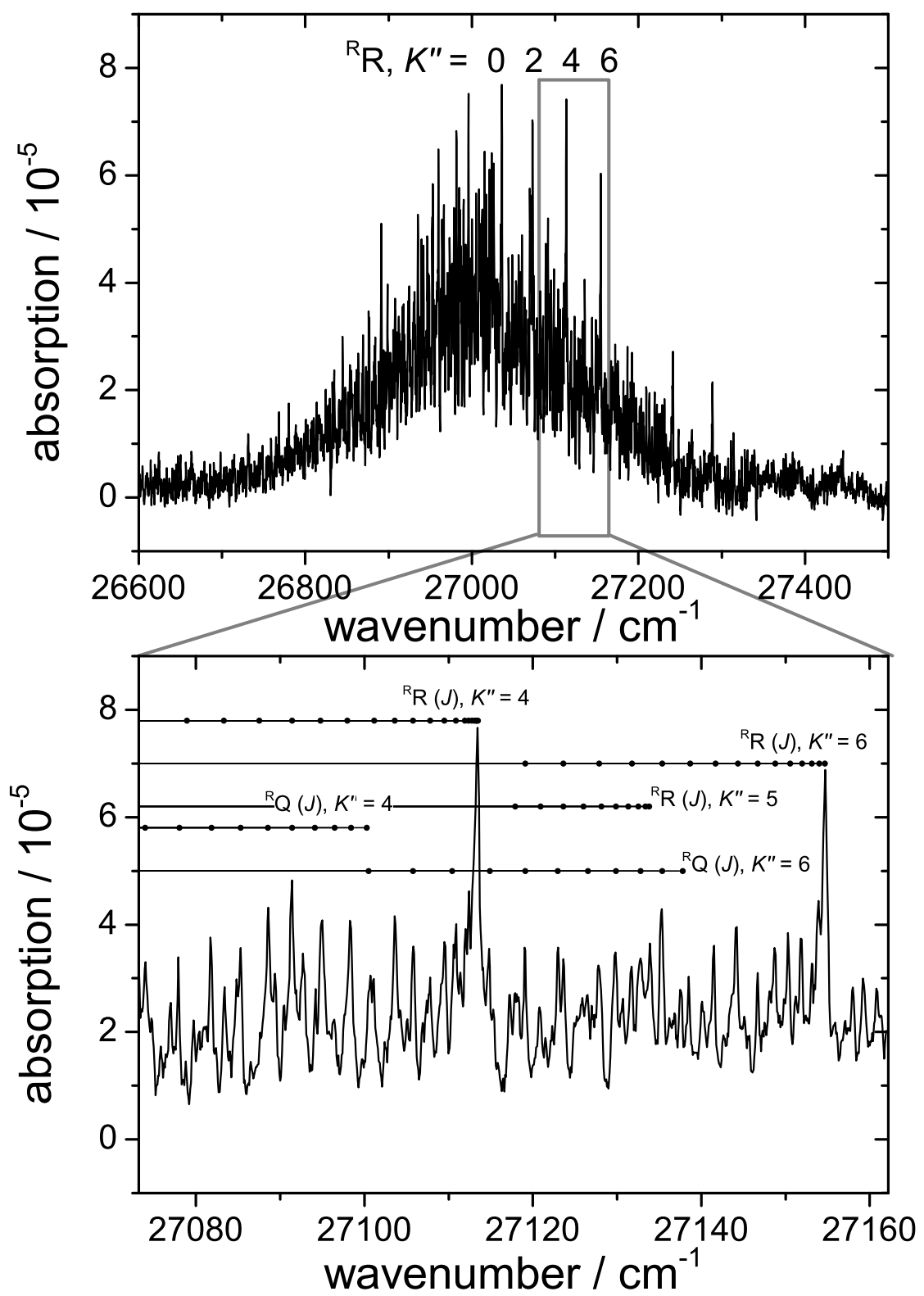

Fig. 1 Top: UV absorption spectrum of the $A-X 4_{1}^{0}$ band of formaldehyde in a fuel rich $(\phi=1.8)$ methane/oxygen/argon flame at 50 mbar $2 \mathrm{~mm}$ above burner surface. Band heads with even $K$ are labeled. Bottom: High resolution spectrum measured in the same flame in the vicinity of the $K^{\prime \prime}=4$ and $K^{\prime \prime}=6$ band heads. Transitions have been assigned according to Dyne[26]. 


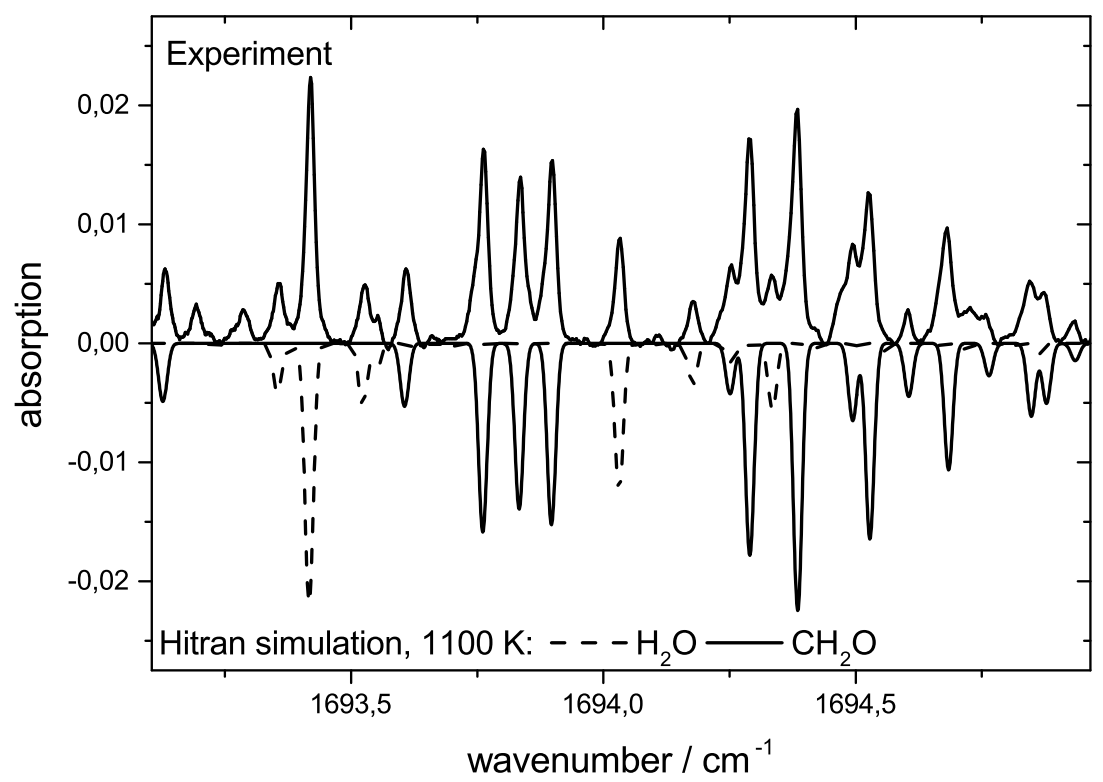

Fig. 2 MIR absorption spectrum of the $\nu_{4}$ band of formaldehyde and water in a fuel rich $(\phi=1.86)$ dimethylether/oxygen/argon flame $1.7 \mathrm{~mm}$ above burner surface. A simulated spectrum based on HITRAN/HITEMP[21,29] is shown on an inverted scale for comparison.

Because of the difficulties associated with quantitative concentration measurements of polyatomic molecules like formaldehyde in the UV we tested in situ MIR absorption spectroscopy as an alternative. A disadvantage in comparison to the measurements in the UV reported above is that only a small spectral region in the MIR can be covered by diode laser based light sources. A MIR absorption spectrum in a fuel rich $(\phi=1.86)$ dimethylether/oxygen/argon flame around $1694 \mathrm{~cm}^{-1}$ is shown in Fig 2. Lines of formaldehyde and water can clearly be identified. Absorption from cold atmospheric water is not a problem in this spectral window, therefore purging the beam path with dry nitrogen is not mandatory. An excellent agreement between measurement and simulated spectrum based on the HITRAN/HITEMP database can be achieved and almost every line can be assigned. In contrast to the measurements in the UV, no broadband absorption background is visible. Additionally, the narrower linewidth helps to avoid interferences with resonances of other molecules.

The intra-pulsed spectroscopy[30] of the QCL used in this work allows to measure a complete absorption spectrum within a single laser pulse at repetition rates up to $100 \mathrm{kHz}$. This is a significant advantage compared to the more common setups involving cw lasers, like the recent work of Wang et al. who used a DFG laser for formaldehyde detection within a shock tube at $3.6 \mu \mathrm{m}$. A two-color scheme has been applied in this work to compensate 
for broadband absorption from interfering species. However, acquisition of a complete absorption spectrum allows simultaneous detection of several formaldehyde lines in a single measurement. Therefore, even narrow-band interferences can easily be identified and taken into account, which is generally not possible with a two-color experiment.

It is interesting to compare the sensitivity for formaldehyde concentration measurements of both techniques used in the work, UV-CRDS and MIR-QCL direct absorption spectroscopy. The minimum detectable absorption of the CRDS experiment can be calculated from the reflectivity of the mirrors $R=99.95 \%$ obtained from the ring-down time of the empty cavity $\tau_{0}=5000 \mathrm{~ns}$ and the error in the ring-down time $\Delta \tau / \tau_{0}=1 \%$. The error in the ring-down time has been determined from the standard deviation of $\tau$ for several individual decay curves, when averaging over 10 laser pulses (1 s measurement time).

$$
\alpha_{\min }=\sigma N_{\min } d=1-R\left(\Delta \tau / \tau_{0}\right)=5 \times 10^{-6}
$$

For concentration measurements in the UV hot-band, the $K^{\prime \prime}=4$ band head is a good choice. This band head covers the unresolved lines $J=6-8$ and has been used before by Luque et al.[8]. The integrated absorption cross section for this band head is $7.5 \times 10^{-21} \mathrm{~cm} /$ molecule at $1000 \mathrm{~K}[8]$. This results in a minimum detectable mole fraction of $2 \times 10^{-4}$ at $1000 \mathrm{~K}$ and $33.3 \mathrm{mbar}$ (determined from the relaxation time $\tau_{-} 0$ and its standard deviation).

We identified three lines of formaldehyde in the MIR between 1693.76 and 1693.90 to be well suited for concentration measurements, since interference with water lines is negligible. The minimum detectable absorption in the Abel-inverted spectra is determined from the noise level to be around $1 \times 10^{-4} \mathrm{~cm}^{-1}$ at a measurement time of $1 \mathrm{~s}$ (20000 averages, determined from the residuum between the measured and the fitted spectrum). For the low-pressure flames investigated here, this results in a minimum $\mathrm{CH}_{2} \mathrm{O}$ mole fraction of $2 \times 10^{-2}$ at $2000 \mathrm{~K}, 4 \times 10^{-3}$ at $1500 \mathrm{~K}$ and $8 \times 10^{-4}$ at $1000 \mathrm{~K}$ and 33.3 mbar.

A a first glance it seems quite astonishing that the sensitivity of CRDS is only a factor of four higher even though the effective absorption path length is about 2000 times longer for UV-CRDS compared to MIR single-pass absorption. The integrated absorption cross section is almost identical in both spectral regions used (UV at $27113.49 \mathrm{~cm}^{-1}: 7.5 \times 10^{-21} \mathrm{~cm} /$ molecule; MIR at $1693.76 \mathrm{~cm}^{-1}: 9.5 \times 10^{-21} \mathrm{~cm} /$ molecule). However, there is a significant difference in the linewidth of the transitions measured: Using a pulsed QCL laser, a linewidth of $0.01 \mathrm{~cm}^{-1}$ can be reached[34], which is sufficient to resolve the true width of the Doppler-broadened resonances in our lowpressure flames (which is around $0.01 \mathrm{~cm}^{-1}$ in the MIR). In contrast, a linewidth of only $0.3 \mathrm{~cm}^{-1}$ can be reached with typical Nd:YAG/dye laser combinations, which is not sufficient to resolve the Doppler-width of transitions in the UV $\left(\approx 0.1 \mathrm{~cm}^{-1}\right)$. In addition, the higher repetition rate of the QCL $(20 \mathrm{kHz}$, in contrast to the $10 \mathrm{~Hz}$ of the Nd:YAG/dye laser system) 
allows much more averaging at the same measurement time. With a multipass setup, the sensitivity of absorption spectroscopy the MIR might even surpass that of UV-CRDS - with the additional benefit of a less complex and less expensive setup. Another advantage of the MIR spectral range is the higher accuracy of the absorption cross sections available. Their uncertainty is only $5-10 \%$ for the MIR transitions at $296 \mathrm{~K}[29]$, while the error of the oscillator strength in the UV is at least $20 \%$ [8].

Taking into account further factors (mainly uncertainties in the temperature), the total error in the absorption cross section in the MIR is $15 \%$. This is, however, still superior to the 30-35\% error achieved in in the UV[8, 28]. In addition, background absorption which is usually present in the UV has to be carefully taken into account to avoid further systematic errors. For the flames investigated here, a significant, but unstructured broadband absorption has been observed in the UV. This might, however, be different for other fuels or flame conditions. In contrast, no broadband absorption is present in the MIR and the lines are well separated. Therefore, several possible systematic errors associated with UV-LIF are not present when using MIR absorption spectroscopy.

Due to the rapid scanning of the QCL wavelength during the laser pulse, the complete absorption spectrum necessary for data evaluation can be measured within a single laser pulse. In contrast, tuning a dye laser is a comparatively slow process, and the acquisition of a spectrum typically requires several minutes. Therefore, measurement times are one or two orders of magnitude less. This makes QCL absorption measurements an excellent choice for tomographic measurements, where large amounts of absorption spectra have to be measured.

It is a well-known fact that tomographic reconstruction is necessary to obtain a reliable flame center concentration profile especially in case of stable polyatomic molecules such as formaldehyde[31,8]. As demonstration, we used the QCL for concentration measurements in a fuel-rich $(\phi=1.86)$ dimethylether/oxygen/argon flame at $33.3 \mathrm{mbar}$. Flame center concentrations were obtained by averaging the Abel-inverted spectra in the homogeneous region up to $15 \mathrm{~mm}$ from the flame center. Model calculation were performed using cantera[32] and the combustion model of Zhao et al.[33]. The temperature profile was measured using $\mathrm{CO}$ and $\mathrm{CO}_{2}$ lines at $4.48 \mu \mathrm{m}$ with a QCL[25]. An error of $5 \%$ is estimated for the temperature. As can be seen from Fig. 3, an excellent agreement between model and experiment was obtained.

\section{Conclusions}

Two different absorption techniques for the detection of formaldehyde in one-dimensional, laminar low-pressure flames have been compared. UVCRDS at $370 \mathrm{~nm}$ has been used to detect formaldehyde in the $A-X 4_{1}^{0}$ band. As a novel alternative method, direct laser absorption spectroscopy of QCL 


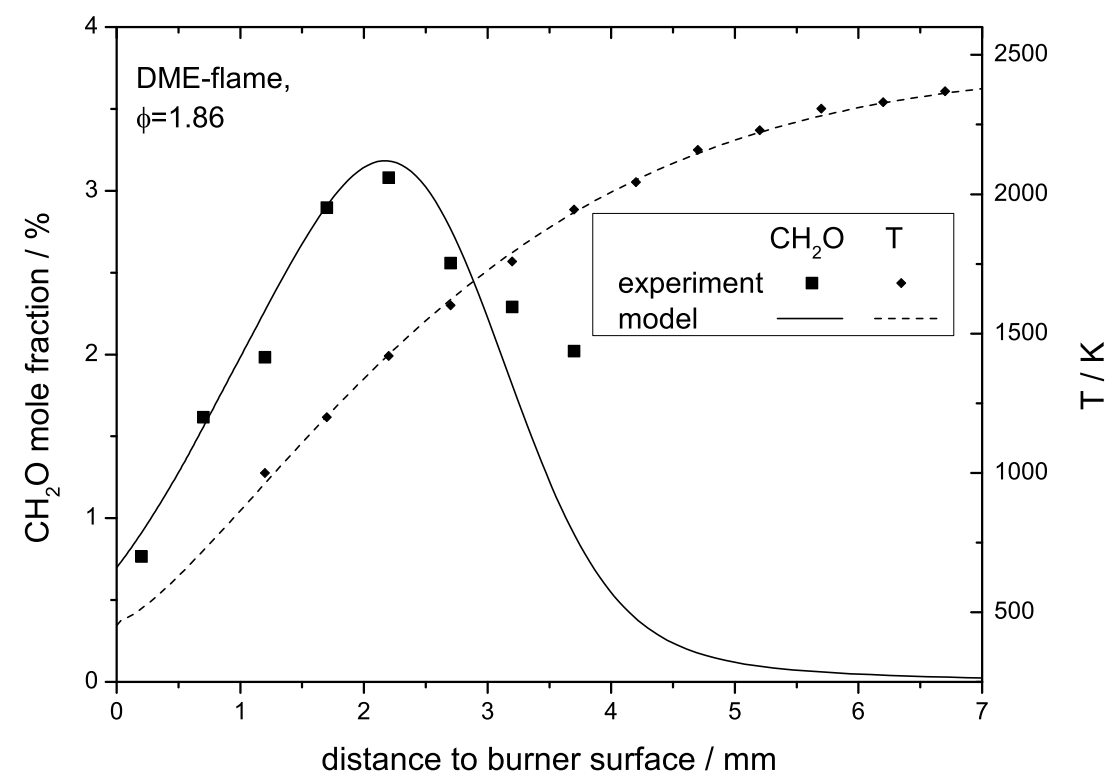

Fig. 3 Flame center concentration profile of $\mathrm{CH}_{2} \mathrm{O}$ measured with MIR absorption spectroscopy in a fuel-rich dimethylether flame $(\phi=1.86)$ compared to model calculations[33].

radiation in the MIR $(5.9 \mu \mathrm{m})$ in the $\nu_{2}$ fundamental band has been used. QCL-LAS has several advantages compared to UV-CRDS: Higher spectral resolution, no broadband background, higher accuracy of the absorption cross section and shorter measurement time. The only major drawback of the present single-pass setup is the reduced effective path length, which is a factor of 2,000 times higher for the highly sensitive multi-pass absorption technique CRDS. Nevertheless, it turns out, that the sensitivity of the CRDS setup is only four times higher that that of the QCL-LAS setup. Major reason for this is the narrower linewidth in the MIR and the higher repetition rate of the QCL which allows more averaging in a given amount of time.

Spatially-resolved formaldehyde measurements in a fuel-rich dimethylether flame have been compared to model calculations, and good agreement has been found both for the absolute concentrations and for the shape of the profile. It has been demonstrated that QCL laser absorption spectroscopy is a valuable tool for flame diagnostics. Especially for the concentration determination of species difficult to detect in the $\mathrm{UV} / \mathrm{Vis}$ (such as stable and/or polyatomic molecules), MIR absorption is a powerful alternative with high potential for the rapid and sensitive detection. 
Acknowledgements This research was funded by in part by DFG in SFB 686 TP B3 and TP C5. The authors wish to thank K. Kohse-Höinghaus for her support and helpful discussions.

\section{References}

1. K. Kohse-Höinghaus, P. Osswald, T. A. Cool, T. Kasper, N. Hansen, F. Qi, C. K. Westbrook and P. R. Westmoreland, Angew. Chem. Int. Ed. 49 (2010) (21), 3572-3597.

2. A. Burkert, D. Grebner, D. Müller, W. Triebel and J. König, Symp. (Int.) Combust. 28 (2000)(2), 1655-1661.

3. S. Böckle, J. Kazenwadel, T. Kunzelmann, D.-I. Shin and C. Schulz, Appl. Phys. B: Lasers Opt. 70 (2000)(5), 733-735.

4. C. S. McEnally and L. D. Pfefferle, Combust. Flame 121 (2000)(4), 575-592.

5. R. Bombach and B. Käppeli, Appl. Phys. B: Lasers Opt. 68 (1999)(2), 251255.

6. P. H. Paul and H. N. Najm, Symp. (Int.) Combust. 27 (1998)(1), 43-50.

7. J. E. Harrington and K. C. Smyth, Chem. Phys. Lett. 202 (1993)(3-4), 196202.

8. J. Luque, J. Jeffries, G. Smith and D. Crosley, Appl. Phys. B: Lasers Opt. 73 (2001) (7), 731-738.

9. R. J. H. Klein-Douwel, J. Luque, J. B. Jeffries, G. P. Smith and D. R. Crosley, Appl. Opt. 39 (2000)(21), 3712-3715.

10. J. Wang, U. Struckmeier, B. Yang, T. A. Cool, P. Osswald, K. KohseHöinghaus, T. Kasper, N. Hansen and P. R. Westmoreland, J. Phys. Chem. A 112 (2008)(39), 9255-9265.

11. M. P. Tolocka and J. H. Miller, Symposium (International) on Combustion 27 (1998)(1), 633-640.

12. U. Struckmeier, P. Osswald, T. Kasper, L. Böhling, M. Heusing, M. Köhler, A. Brockhinke and K. Kohse-Höinghaus, Z. Phys. Chem. 223 (2009), 503-537.

13. S. Wang, D. F. Davidson and R. K. Hanson, Combust. Flame 160 (2013)(10), 1930-1938.

14. S. C. Herndon, M. S. Zahniser, J. Nelson, David D., J. Shorter, J. B. McManus, R. Jiménez, C. Warneke and J. A. de Gouw, J. Geophys. Res. 112 (2007), D10S03, 15 PP.

15. A. Elia, C. Di Franco, V. Spagnolo, P. M. Lugar and G. Scamarcio, Sensors 9 (2009)(4), 2697-2705.

16. A. Cheesman, J. A. Smith, M. N. R. Ashfold, N. Langford, S. Wright and G. Duxbury, J. Phys. Chem. A 110 (2006), 2821-2828.

17. W. Ren, A. Farooq, D. Davidson and R. Hanson, Appl. Phys. B: Lasers Opt. 107 (2012), 849-860.

18. S. D. Wehe, M. G. Allen, X. Liu, J. Jeffries and R. Hanson, NO and CO Absorption Measurements with a Mid-IR Quantum Cascade Laser for Engine Exhaust Applications, in paper AIAA 2003-0588 at 41st Aerospace Sciences Meeting, Reno, NV, Jan. 2003 (2003).

19. J. Vanderover and M. A. Oehlschlaeger, Appl. Phys. B: Lasers Opt. 99 (2010)(1-2), 353-362.

20. HITRAN database, www.cfa.harvard.edu/HITRAN/. 
21. L. S. Rothman, I. E. Gordon, R. J. Barber, H. Dothe, R. R. Gamache, A. Goldman, V. Perevalov, S. A. Tashkun and J. Tennyson, J. Quant. Spectrosc. and Rad. Transfer 111 (2010), 2139-2150.

22. C. J. Dasch, Appl. Opt. 31 (1992)(8), 1146-1152.

23. R. Villarreal and P. Varghese, Appl. Opt. 44 (2005)(31), 6786-6795.

24. J. Humlicek, J. Quant. Spectrosc. Radiat. Transfer 27 (1982)(4), 437-444.

25. P. Nau, J. Koppmann, A. Lackner, K. Kohse-Höinghaus, A. Brockhinke "Quantum cascade laser based MIR spectrometer for the determination of $\mathrm{CO}$ and $\mathrm{CO}_{2}$ concentration and temperature in flames"' Submitted for publication in Appl. Phys. B (2014)

26. P. J. Dyne, J. Chem. Phys. 20 (1952)(5), 811-818.

27. D. Shin, G. Peiter, T. Dreier, H.-R. Volpp and J. Wolfrum, Symp. (Int.) Combust. 28 (2000)(1), 319-325.

28. D. Shin, T. Dreier and J. Wolfrum, Appl. Phys. B: Lasers Opt. 72 (2001)(2), 257-261.

29. L. Rothman, I. Gordon, A. Barbe, D. Benner, P. Bernath, M. Birk, V. Boudon, L. Brown, A. Campargue, J.-P. Champion, K. Chance, L. Coudert, V. Dana, V. Devi, S. Fally, J.-M. Flaud, R. Gamache, A. Goldman, D. Jacquemart, I. Kleiner, N. Lacome, W. Lafferty, J.-Y. Mandin, S. Massie, S. Mikhailenko, C. Miller, N. Moazzen-Ahmadi, O. Naumenko, A. Nikitin, J. Orphal, V. Perevalov, A. Perrin, A. Predoi-Cross, C. Rinsland, M. Rotger, M. Šimečková, M. Smith, K. Sung, S. Tashkun, J. Tennyson, R. Toth, A. Vandaele and J. V. Auwera, J. Quant. Spectrosc. Radiat. Transfer 110 (2009), $533-572$.

30. E. Normand, M. McCulloch, G. Duxbury and N. Langford, Opt. Lett. 28 (2003), 16-18.

31. P. Nau, A. Seipel, A. Lucassen, A. Brockhinke and K. Kohse-Höinghaus, Experiments in Fluids 49 (2010)(9), 761-773.

32. Cantera 2.0.0b3, http://code.google.com/p/cantera/ (2012).

33. Z. Zhao, M. Chaos, A. Kazakov and F. L. Dryer, Int. J. Chem. Kinet. 40 (2008)(1), 1-18.

34. T. Beyer, M. Braun, S. Hartwig and A. Lambrecht, J. Appl. Phys. 95 (2004)(9), 4551-4554. 